\title{
Tetrandrine suppresses metastatic phenotype of prostate cancer cells by regulating Akt/mTOR/MMP-9 signaling pathway
}

\author{
BO KOU ${ }^{1^{*}}$, WEI LIU ${ }^{2 *}$, WENBO HE $^{3 *}$, YUANYUAN ZHANG ${ }^{4}$, JIANJIE ZHENG $^{1}$, \\ YANG YAN $^{1}$, YONGJIAN ZHANG ${ }^{1}$, SUOCHUN XU $^{1}$ and HAICHEN WANG ${ }^{1}$
}

\author{
Departments of ${ }^{1}$ Cadiovascular Sugery and ${ }^{2}$ Urology, First Affiliated Hospital of Medical School, \\ Xi'an Jiaotong University; ${ }^{3}$ Medical School, Xi'an Jiaotong University; ${ }^{4}$ Department of Burn and \\ Plastic Surgery, Xi'an Central Hospital, Xi'an, Shaanxi 710061, P.R. China
}

Received October 19, 2015; Accepted November 24, 2015

DOI: $10.3892 / o r .2016 .4649$

\begin{abstract}
Tetrandrine (TET), a bisbenzylisoquinoline alkaloid found in traditional Chinese medicines, exerts anticancer activity in vitro and in vivo. However, its potential role in the prostate cancer metastatic process has not yet been elucidated. Thus, we investigated the inhibition effect of tetrandrine on prostate cancer migration and invasion and the corresponding molecular basis underlying its anticancer activity. Cell migration and invasion were determined using the Transwell chamber model. The protein expression of Akt, phosphorylated Akt, the mammalian target of rapamycin (mTOR), phosphorylated mTOR and matrix metalloproteinases 9 (MMP-9) was detected by western blot in the presence or absence of tetrandrine or in the group tetrandrine combination with LY294002 (inhibitor of Akt) and rapamycin (inhibitor of mTOR). Our studies showed that excluding the effect of tetrandrine on cell proliferation, tetrandrine significantly inhibited cell migration and invasion in prostate cancer DU145 and PC3 cells. Furthermore, tetrandrine decreased the protein levels of p-Akt, p-mTOR, and MMP-9. While the inhibition of Akt or mTOR by the respective inhibitors could potentiate this effect of tetrandrine on prostate cancer cells, the studies indicate that tetrandrine inhibits the metastasis process by negatively regulating the Akt/mTOR/MMP-9 signaling pathway. These results suggest that tetrandrine might serve as a potential metastasis suppressor to treat cancer cells that have escaped surgical removal or that have disseminated widely.
\end{abstract}

Correspondence to: Dr Suochun $\mathrm{Xu}$ or Dr Haichen Wang, Department of Cadiovascular Sugery, First Affiliated Hospital of Medical School, Xi'an Jiaotong University, \#277 Yanta West Road, Xi'an, Shaanxi 710061, P.R China

E-mail: xusuochun@163.com

E-mail:whccvsdc@126.com

*Contributed equally

Key words: tetrandrine, prostate cancer, migration, invasion, Akt/mTOR/MMP-9 signaling

\section{Introduction}

Prostate cancer ( $\mathrm{PCa})$, one of the most prevalent malignancies in male patients worldwide, causes considerable morbidity and mortality, and a great deal of attention is currently given to its tumorigenesis. Androgen signaling based on the androgen receptor is an essential oncogenic pathway for PCa progression (1) and deprivation of androgen is used widely as the basic therapeutic strategy for patients with androgen-dependent PCa (2). Nevertheless, most patients have a recurrence with a more aggressive form, known as castration-resistant prostate cancer (CRPC) (3), with $80 \%$ of CRPC patients experiencing the presence of bony metastases. Due to its frequent metastasis and significant heterogeneity, CRPC is difficult to cure, and it has become a vexing problem for the oncologists and clinicians (4). Therefore, it is of vital importance to identify appropriate agents to kill selectively or sensitize prostate cancer.

Tetrandrine (TET) is a bisbenzylisoquinoline alkaloid with the molecular structural formula $\mathrm{C}_{38} \mathrm{H}_{42} \mathrm{~N}_{2} \mathrm{O}_{6}$ and a molecular weight of $622.74988 \mathrm{~g} / \mathrm{mol}$. Tetrandrine isolated from the Chinese herbal medicine Stephaaniae has been applied to a very broad spectrum of pharmacological events (5), such as antihypertension, antiarrhythmia, and antirheumatism. In recent decades, tetrandrine has been used as an antifibrotic agent in the treatment of silicosis (6), and accumulating evidence suggests that tetrandrine exerts strong anticancer effects on diverse cancers in vitro, including colon $(7,8)$, hepatoma (9), bladder (10), and lung cancer (11). The beneficial impact of tetrandrine on tumor cell multidrug resistance (12), radiosensitization (13), and angiogenesis (14) has also attracted a great deal of attention. Also, tetrandrine has been shown to modulate multiple cellular signaling events, including the Wnt $/ \beta$ signaling pathway (15), mitogen-activated protein kinase activation (16), and NF- $\mathrm{KB}$ signaling pathway.

In our previous study, we found that tetrandrine exhibited anticancer effects against $\mathrm{PCa}$ in vitro by suppressing cell proliferation, inducing apoptosis and inhibiting cell migration and invasion. Despite its potential as an anticancer constituent, the underlying mechanism of tetrandrine on PCa metastasis has not yet been elucidated. Therefore, we investigated the possible mechanism of tetrandrine on the inhibitory effect of metastasis in PCa DU145 and PC-3 cells. 


\section{Materials and methods}

Cell culture. Human PCa cell lines DU145 and PC-3 were obtained from the American Type Culture Collection (Manassas, VA, USA). The cells were cultured in Dulbecco's modified Eagle's medium/1640 supplemented with 10\% fetal bovine serum (Gibco, Grand Island, NY, USA) and $1 \%$ penicillin-streptomycin (Invitrogen, Carlsbad, CA, USA), in a humidified atmosphere with $5 \% \mathrm{CO}_{2}$ at $37^{\circ} \mathrm{C}$.

Reagents. Tetrandrine $\left(\mathrm{C}_{38} \mathrm{H}_{42} \mathrm{~N}_{2} \mathrm{O}_{6}\right)$ and 3-(4,5-dimethylthiazol-2-yl)-2,5-diphenyltetrazolium bromide (MTT) were purchased from Sigma Chemical Co. (St. Louis, MO, USA). Tetrandrine was diluted with $0.1 \mathrm{~mol} / 1 \mathrm{HCl}$ at a concentration of $25 \mathrm{mg} / \mathrm{ml}^{-1}$, then added to the cell culture supernatant in appropriate proportions. Antibodies against Akt, phosphoAkt, the mammalian target of rapamycin (mTOR), matrix metalloproteinase-9 (MMP-9) and peroxidase-conjugated secondary antibodies were obtained from Cell Signaling Technology, Inc. (Beverly, MA, USA). LY294002 (Akt inhibitor) and rapamycin (mTOR inhibitor) were obtained from Santa Cruz Biotechnology, Inc. (Santa Cruz, CA, USA). The enhanced chemiluminescence (ECL) detection system was obtained from Amersham Life Science, Inc. (Arlington Heights, IL, USA).

MTT assay. A modified MTT assay was used to assess cell proliferation viability. Briefly, DU145 and PC-3 cells were seeded in 96 -well plates $\left(8 \times 10^{3}\right.$ cells/well, $90 \%$ density) and incubated in the presence or absence of tetrandrine for various periods of time. Then, $0.5 \mathrm{mg} / \mathrm{ml} \mathrm{MTT}$ dye solution was added to each well and incubated at $37^{\circ} \mathrm{C}$ for $4 \mathrm{~h}$. After incubation, the culture medium was discarded and the cells were lysed with dimethyl sulfoxide to dissolve the formazan crystals. Absorbance at a wavelength of $490 \mathrm{~nm}$ was detected using a 96-well microplate reader (Bio-Rad, Hercules, CA, USA). The experiments were performed in triplicate.

Transwell migration assay. Transwell migration assays were performed using PCa DU145 and PC3 cells after treatment with tetrandrine. Cells (DU145: 6x104 or PC-3: $5 \times 10^{4}$ in $200 \mu 1$ medium of serum starvation, respectively) were seeded into the top chamber, and $800 \mu \mathrm{l}$ of medium supplemented with $10 \%$ fetal calf serum were added to the lower chamber. After incubation at $37^{\circ} \mathrm{C}$ for various times, cells adhering to the top chambers were removed with a cotton swab. The migratory cells on the lower surface of the membrane were fixed with $4 \%$ paraformaldehyde and stained with $0.1 \%$ crystal violet (Beyotime, Shanghai, China). Cells that migrated to the lower surface were counted in five randomly chosen visual fields under a microscope at $\times 100$ magnification. The data were obtained from three independent experiments.

Matrigel invasion assay. The Transwell chambers (polycarbonic membrane, $6.5-\mathrm{mm}$ diameter, $8 \mu \mathrm{m}$ pore size) were coated with $50 \mu 1$ Matrigel (Matrigel:serum-free medium 1:5). After incubation at $37^{\circ} \mathrm{C}$ for five hours, the cells (DU145: $10 \times 10^{4}$ or PC-3: $12 \times 10^{4}$ in $200 \mu$ medium of serum starvation, respectively) were treated according to the protocol procedure, which was similar to the Transwell migration assay.
Western blotting. The PCa cells were harvested $24 \mathrm{~h}$ after the tetrandrine treatment, and the total cell lysates were denatured with lysis buffer $[10 \mathrm{mmol} / \mathrm{l}$ Tris- $\mathrm{HCl}(\mathrm{pH} 7.4), 150 \mathrm{mmol} / \mathrm{l}$ $\mathrm{NaCl}, 0.1 \%$ sodium dodecyl sulfate (SDS), $1 \mathrm{mmol} / \mathrm{l}^{-1}$ ethylenediaminetetraacetic acid, $1 \mathrm{mmol} / \mathrm{l}$ ethylene glycol tetraacetic acid, $0.3 \mathrm{mmol} / 1$ phenylmethylsulfonyl fluoride, $0.2 \mathrm{mmol} / 1$ sodium orthovanadate, $1 \% \mathrm{NP}-40,10 \mathrm{mg} / \mathrm{ml}$ leupeptin, and $10 \mathrm{mg} / \mathrm{ml}$ aprotinin]. Then clarified protein lysates ( 30-60 $\mu \mathrm{g}$ whole-cell lysates, mitochondrial and cytosolic fractions) were resolved electrophoretically on denaturing SDS-polyacrylamide gel (10\%) and transferred to nitrocellulose membranes. Immunoblotting was performed with the primary antibody, anti-MMP-9, as well as anti-mTOR, and p-Akt overnight at $4^{\circ} \mathrm{C}$. The membranes were washed and incubated with peroxidase-conjugated secondary antibody at room temperature $\left(25^{\circ} \mathrm{C}\right)$. Ultimately, the proteins of interest were visualized with ECL Substrate and exposed to X-ray film.

Gelatin zymography. Gelatin zymography was performed following the standardized protocol (17). Briefly, $5 \times 10^{5}$ cells were treated with tetrandrine for $24 \mathrm{~h}$, after which the supernatants were collected to load onto $10 \%$ polyacrylamide gels and co-polymerized with $0.1 \%$ gelatin (Sigma). After undergoing electrophoresis, the gels were washed twice for $40 \mathrm{~min}$ in $2.5 \%$ Triton X-100 and $50 \mathrm{mmol} / \mathrm{l}$ Tris- $\mathrm{HCl}$. Then, the gels were incubated for the next $24 \mathrm{~h}$ at $37^{\circ} \mathrm{C}$ in buffer containing $50 \mathrm{mmol} / \mathrm{l}$ Tris- $\mathrm{HCl}, 5 \mathrm{mmol} / 1 \mathrm{CaCl}_{2}$ and $0.02 \% \mathrm{NaN}_{3}$, followed by staining with Coomassie brilliant G-250 and destaining with $20 \%$ methanol and $10 \%$ acetic acid. Finally, the gels were visualized with a molecular imager (ChemiDoc XRS+, Bio-Rad).

Statistical analysis. SPSS 17.0 software (SPSS Inc., Chicago, IL, USA) was used for statistical analyses. Statistical differences between the vehicle and drug treatment groups were compared by one-way analysis of variance, and Dunnett's t-test was used for multiple comparisons. Student's t-test (two-sided) was used for comparisons involving only two groups. A value of $\mathrm{P}<0.05$ was considered statistically significant.

\section{Results}

Effects of tetrandrine on cell migration and invasion in prostate cancer DU145 and PC-3 cells. The MTT assays showed that cell proliferation was significantly inhibited by tetrandrine at a concentration of $\geq 2.5 \mu \mathrm{M}$ in a cell density $>90 \%$ (Fig. 1). In view of these results, tetrandrine at $2.5 \mu \mathrm{M}(\mathrm{a}<10 \%$ inhibitory rate) was chosen as the representative dose in the subsequent in vitro studies, to exclude the suppressing interference from $\mathrm{PCa}$ proliferation by tetrandrine.

To determine whether tetrandrine regulates cellular metastatic processes, we detected the effects of tetrandrine on $\mathrm{PCa}$ cell migration. Using a Transwell migration assay, we found that tetrandrine functioning in the DU145 cell line decreased cell migration approximately 2.5 -fold after $24 \mathrm{~h}$ (Fig. 2A and C). Similarly, treatment of the PC-3 cells with tetrandrine resulted in a greater than 2.8 -fold decrease in migration compared to the control group (Fig. 2B and D).

Next, using a Matrigel invasion assay, we explored whether tetrandrine could affect the invasiveness of $\mathrm{PCa}$ 
A

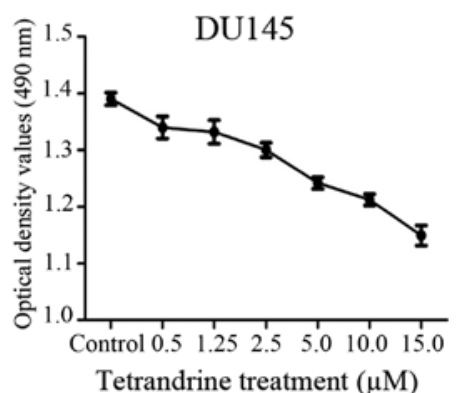

C

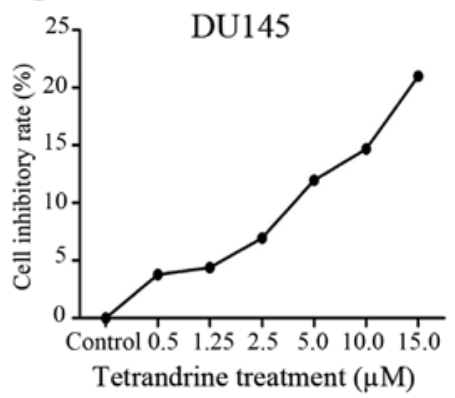

B

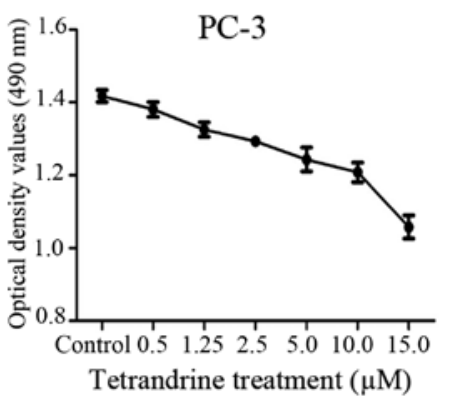

D

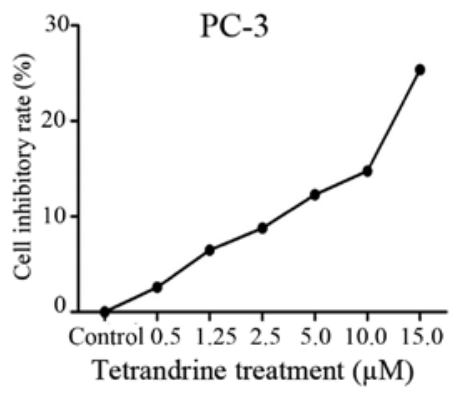

Figure 1. Tetrandrine decreases prostate cancer cell proliferation. The proliferation of cells $<90 \%$ cell density with various doses of tetrandrine was compared to that of negative-control cells. The mean cell number ( \pm SEM) of three independent experiments performed in triplicate is shown for DU145 (A and C) and PC-3 (B and D) cells.
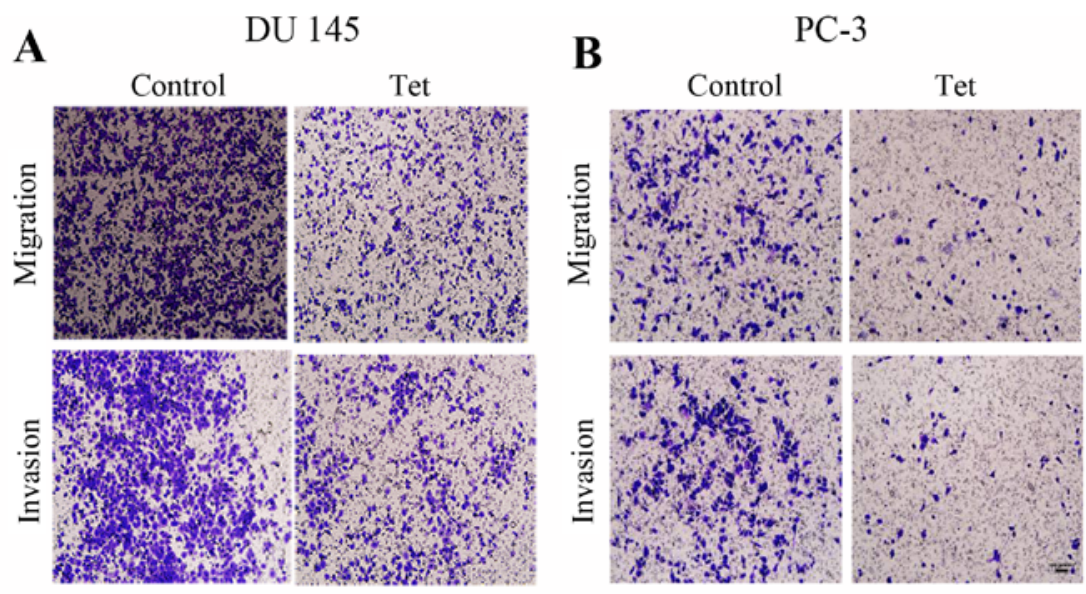

\section{C}

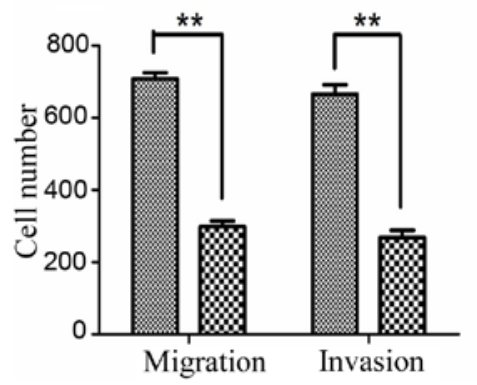

\section{D}

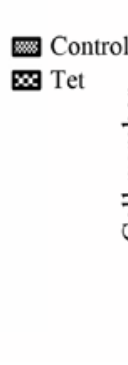

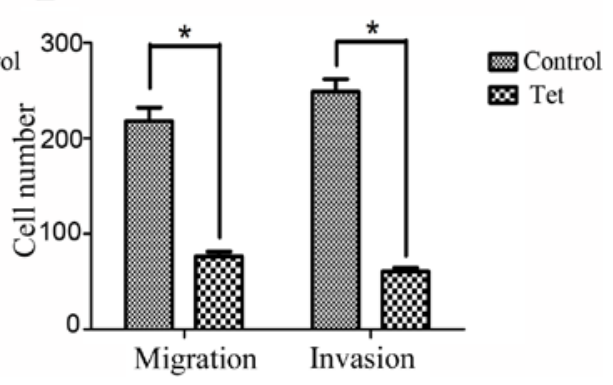

Figure 2. Tetrandrine decreases cell migration and invasiveness in prostate cancer. Transwell invasion assay and Matrigel invasion assay were performed on DU145 (A and C) and PC-3 (B and D) cells treated with tetrandrine. Upper panels show representative regions of the chamber filters with crystal violet-stained cells. The fold change represents the number of migrated or invaded cells per chamber divided by controls from three independent experiments performed in triplicate $(\mathrm{P}<0.05)$.

cells. Serum-starved cells were added to the upper chambers of the Transwell and the total number of cells invading through the Matrigel barrier in response to a chemoattractant (serum) at various times were counted. Tetrandrine significantly decreased the number of invading cells by $>2.4$-fold and 4 -fold in the DU145 and PC-3 cells, respectively (Fig. 2). 
A

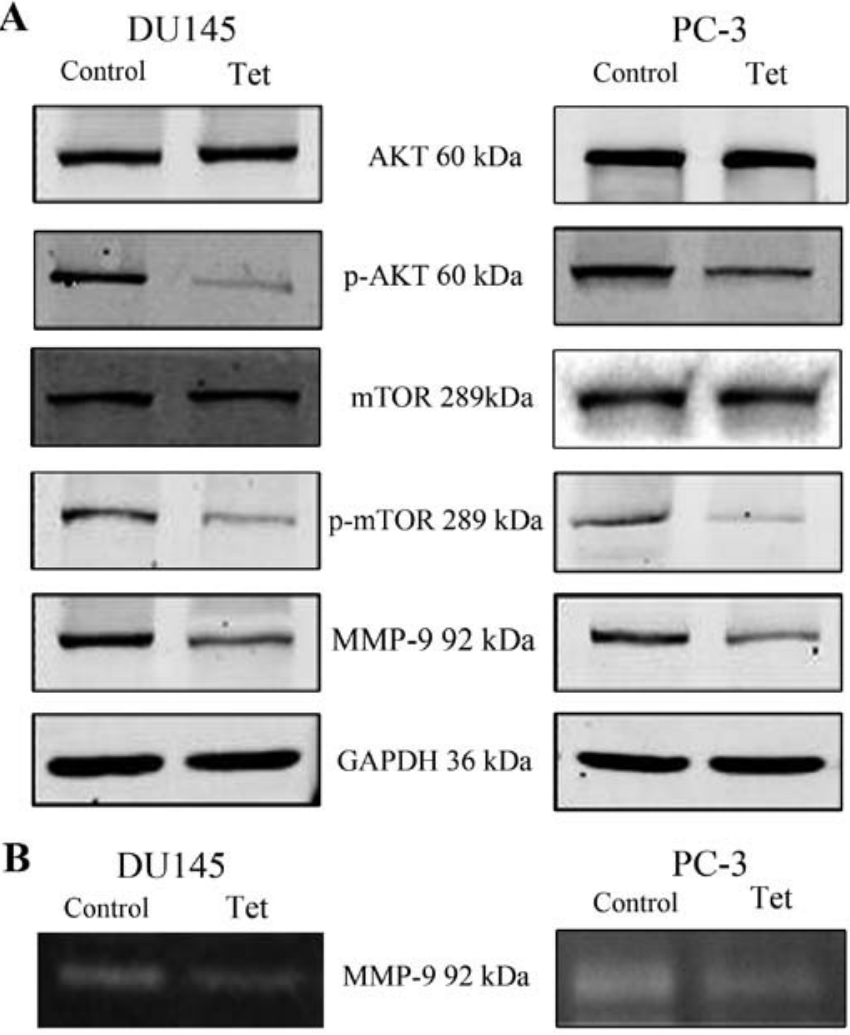

Figure 3. Tetrandrine significantly reduces phospho-AKT, phospho-mTOR and MMP-9 expression and decreases MMP-9 activity in prostate cancer cell lines. (A) DU145 or PC3 cells with tetrandrine treatment were subjected to western blotting for AKT, phospho-AKT, mTOR, phospho-mTOR and MMP-9. GAPDH was used as a loading control. Representative blots from three experiments are shown. (B) Representative gelatin zymography of the enzyme activity of MMP-9 in the supernatants of DU145 and PC-3 exposed to tetrandrine. The experiments were performed in triplicate.

These results suggest that tetrandrine might play a vital role in inhibiting the migration and invasion potential of human PCa cells, as indicated by the observation in the Transwell migration and Matrigel invasion assays.

Identification of the signaling pathway regulated by tetrandrine in prostate cancer. To define the underlying mechanism of tetrandrine-mediated inhibition of metastatic processes in $\mathrm{PCa}$, we investigated the expression of several key factors in cancer cell migration and invasion, including E-cadherin, $\mathrm{N}$-cadherin, vimentin, and MMP-9. To determine the impact of tetrandrine on the production of proteinases by DU145 and PC-3, protein lysates were collected and subjected to SDS-polyacrylamide gel electrophoresis. As shown in Fig. 3, the presence of proteinases led to a clear band at $92 \mathrm{kDa}$ in the nitrocellulose membranes, which was assigned to MMP-9, and tetrandrine reduced MMP-9 protein levels (Fig. 3A) and decreased MMP-9 activity (Fig. 3B), which corresponded to the inhibition of invasiveness in the DU145 and PC-3 cells. However, the protein levels of E-cadherin, $\mathrm{N}$-cadherin and vimentin in the tetrandrine treatment remained the same (data not shown).

To detect the upstream of MMP-9, we found that the pretreatment with tetrandrine resulted in a marked decrease in the protein levels of phosphorylated Akt (p-Akt) and phosphorylated mTOR (p-mTOR) in both the DU145 and
PC-3 cells (Fig. 3). To gain further insights into the molecular mechanisms of tetrandrine in the PCa metastasis process, LY294002 (Akt inhibitor) or rapamycin (mTOR inhibitor) was selected to combine with tetrandrine for functional and mechanism research. The data showed that pretreatment with LY294002 decreased the tetrandrine-inhibited migration and invasion in the DU145 and PC-3 cells (Fig. 4), and reduced the p-mTOR and MMP-9 protein levels (Fig. 5) much more significantly compared with the tetrandrine alone group. This result suggests that Akt protein was passably related to the antimetastasis effect of tetrandrine.

Given that Akt protein might contribute to the anticancer activity of tetrandrine, the downstream of Akt was evaluated to define further the molecular basis of tetrandrine-inhibited metastasis. Similar results were observed in a combination of tetrandrine and rapamycin. The combined effect of the two agents was found to suppress prostate cancer cell migration and invasion (Fig. 6), and diminish of MMP-9 protein levels more effectively (Fig. 7); there was no significant change in NF- $\kappa B$ protein level (data not shown).

Taken together, our findings show that the Akt/mTOR/ MMP-9 signaling pathway was partially associated with tetrandrine-inhibited metastasis in CRPC cells, suggesting that tetrandrine might be a potential promising agents in the suppression of metastatic phenotypes in PCa treatment.

\section{Discussion}

In recent decades, the incidence of PCa has increased sharply in Asian countries (18). Until now, the majority of clinical trials have achieved limited benefits in the treatment of advanced PCa, mainly because of the high incidence of invasion and metastasis. Metastasis, a symbol of malignancy, is the migration of cancer cells from the original tumor site to distant organs through the bloodstream or lymph system. Metastasis development is a multi-step process that is implicated in such activities as local invasion, transfer, extravasation, and tumor deposit (19). Shedding light on the mechanisms that facilitate tumor cell migration and invasion is of major concern in cancer research, as approximately $90 \%$ of deaths from solid tumors arise from metastasis. The median survival rate for patients with metastatic PCa is far less than five years while the opposite is true for men with localized disease (19). Based on these factors, it is known that the tendency to invade and transfer is one of the obstacles facing PCa treatments. Hence, there is an urgent need to develop new agents for PCa therapy.

Tetrandrine, a traditional Chinese medicine, has been shown to exhibit a wide range of uses. Accumulating evidence indicates that tetrandrine exerts antitumor effects against various cancer cells in vitro by inducing cell cycle arrest and inhibiting angiogenesis. In our previous study, we found that the induction of PCa cell apoptosis by tetrandrine might be mediated partially by the activation of caspase cascade. Nevertheless, it is conceivable that the anticancer activity of tetrandrine might be involved with many other signaling pathways. It has been reported that tetrandrine induces apoptosis in hepatocellular carcinoma cells by generating reactive oxygen species, followed by the repression of Akt activity (20). Tetrandrine also exhibits anti-proliferation effects by targeting $\beta$-catenin activity to a certain extent. In addition, it has been 


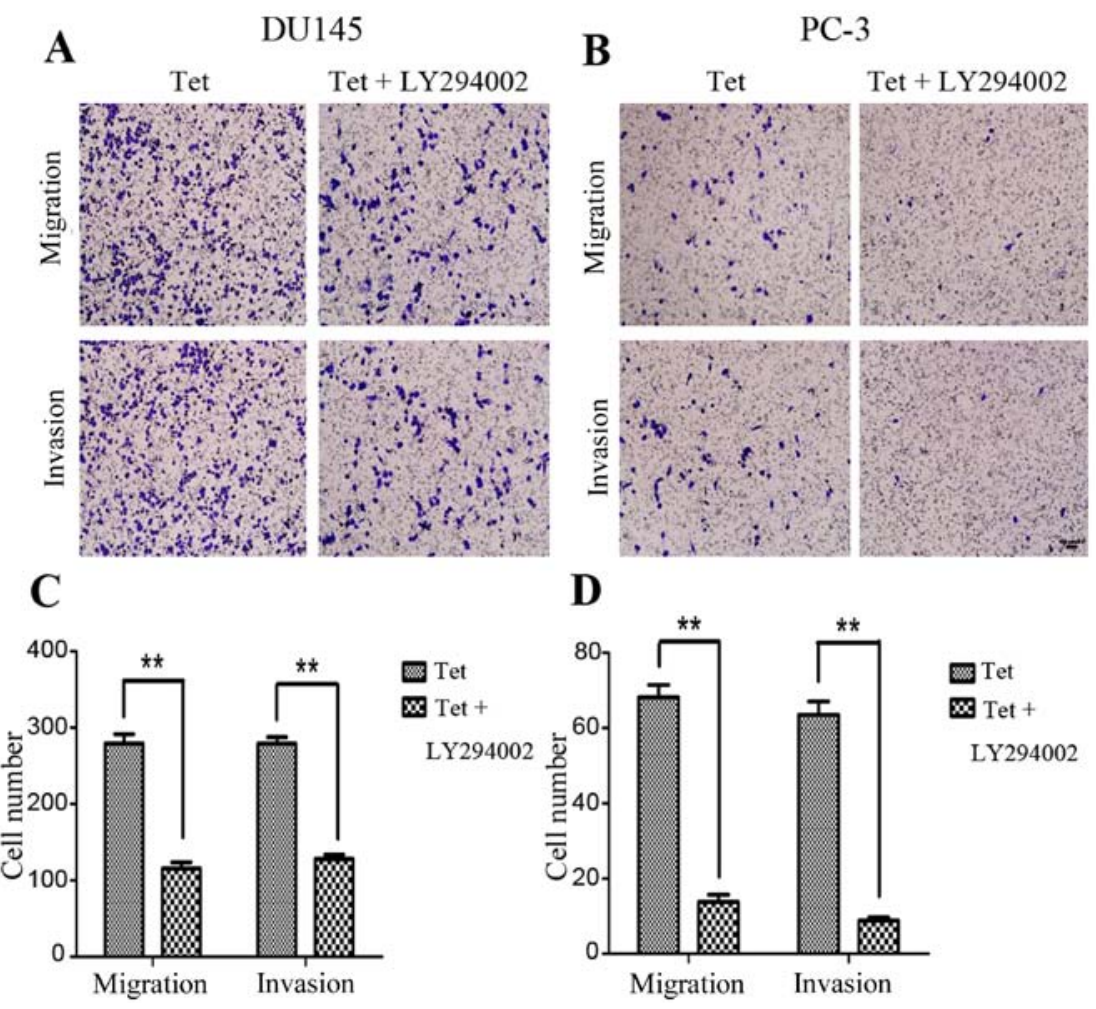

Figure 4. Tetrandrine combined with Akt inhibitor LY294002 significantly inhibits cell migration and invasiveness in castration-resistant prostate cancer cell lines. DU145 (A and C) and PC-3 (B and D) cells that penetrated the membrane were counted after $24 \mathrm{~h}$ as described in Materials and methods. Quantification of three independent experiments is shown with error bars representing SEM.

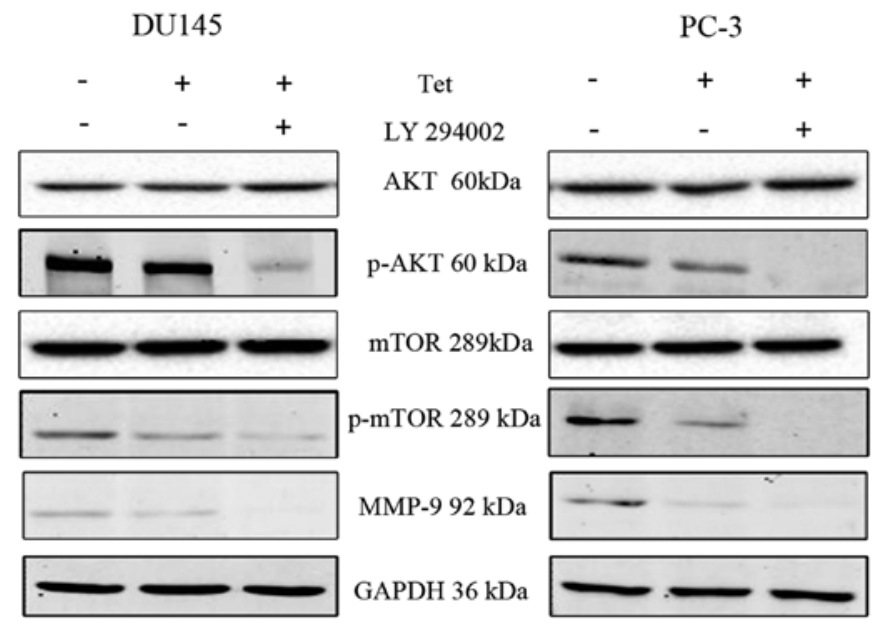

Figure 5. Tetrandrine and Akt inhibitor LY294002 synergistically decreased phospho-mTOR and MMP-9 protein levels more effectively than tetrandrine alone. DU145 and PC-3 cells treated with tetrandrine or LY294002 were subjected to western blotting for phospho-Akt, mTOR, phospho-mTOR, MMP-9, and GAPDH. Representative blots from three experiments are shown.

reported that tetrandrine inhibited in vivo tumor metastasis in a mouse model of stage IV breast cancer. The inhibitory effect of tetrandrine on breast cancer metastasis might be mediated partly by regulating endothelial cell-specific molecule-1 (ESM-1), integrin $\beta 5$ protein and intercellular cell adhesion molecule-1 (ICAM-1) levels (21).

Metastasis in patients with $\mathrm{PCa}$ is one of the main lethal factors blocking direct treatment. In our studies, the impact of tetrandrine resulted in decreased cell migration and invasion in the metastatic CRPC cell lines DU145 and PC-3. Identifying the molecular mechanisms of tetrandrine on the metastatic process underlying $\mathrm{PCa}$ is crucial in order to clarify our understanding of tumorigenesis and metastasis. As such, we have detected the expression of several key factors in the cell metastatic process and found that tetrandrine might decrease MMP-9 protein levels and activity in two independent CRPC cell lines; however, we found no significant changes in MMP-2 protein levels. These findings indicate that MMP-9 deregulation could be a vital event in PCa progression and are consistent with our data that tetrandrine expression resulted in decreased MMP-9 levels and a decline in metastatic traits.

In addition to speculating that MMP-9 might be associated with anti-metastasis activity displayed by tetrandrine, we also measured the Akt signaling pathway, which is upstream of MMP-9. The results showed that p-Akt and p-mTOR protein levels decreased significantly with tetrandrine treatment. Next, to determine whether the ability of tetrandrine to inhibit PCa metastasis was mediated through a decrease in p-Akt and p-mTOR kinase activity, we treated DU145 and PC-3 cells with tetrandrine in combination with LY294002 and rapamycin, respectively, and then documented cell migration and invasion using Transwell assay and western blotting. Our data in the present study demonstrated that the inhibition of metastasis in DU145 and PC3 cells by tetrandrine was markedly enhanced in combination with LY294002 or rapamycin pre-treatment.

Furthermore, tetrandrine and LY294002 synergistically decreased p-mTOR and MMP-9 protein levels, and rapamycin worked in the same manner. However, whether the decrease in p-mTOR protein level is directly related to downregulation of MMP-9 protein needs to be investigated further. These data 
A

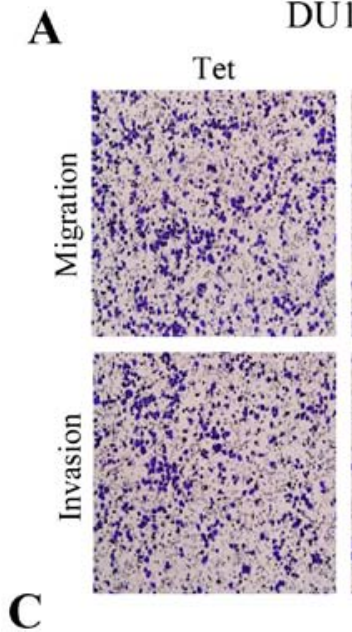

DU145

Tet + rapamycin
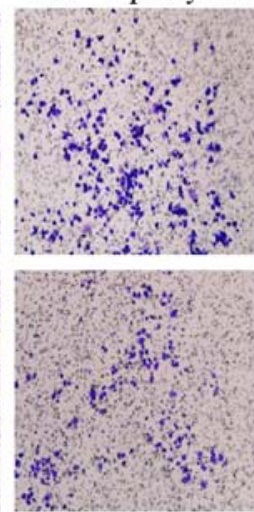

B

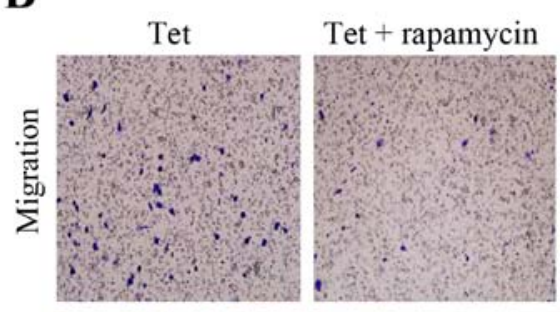

D
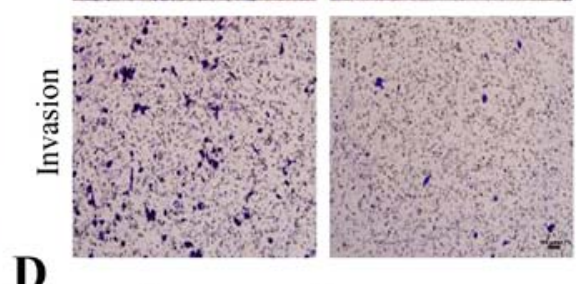
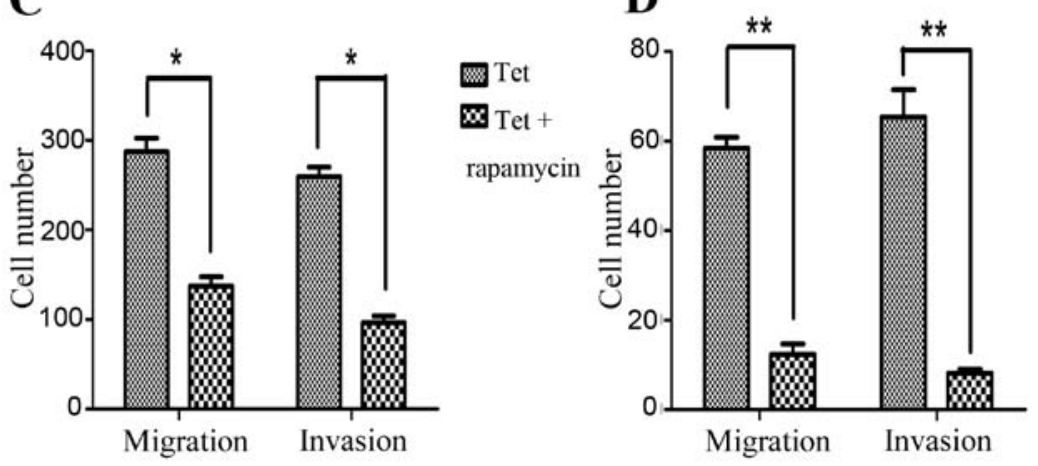

感 Tet

$\mathbf{x} \mathrm{Tet}+$

rapamycin

Figure 6. Pretreatment with mTOR inhibitor rapamycin more significantly decreased tetrandrine-inhibited migration and invasion in castration-resistant prostate cancer cell lines. Transwell migration assay and Matrigel invasion assay were performed on DU145 (A and C) and PC-3 (B and D) cells treated with tetrandrine or rapamycin. Five fields were randomly chosen and visualized by microscopy to evaluate the ability of cell migration and invasiveness. Quantification of three independent experiments is shown, with error bars representing SEM.

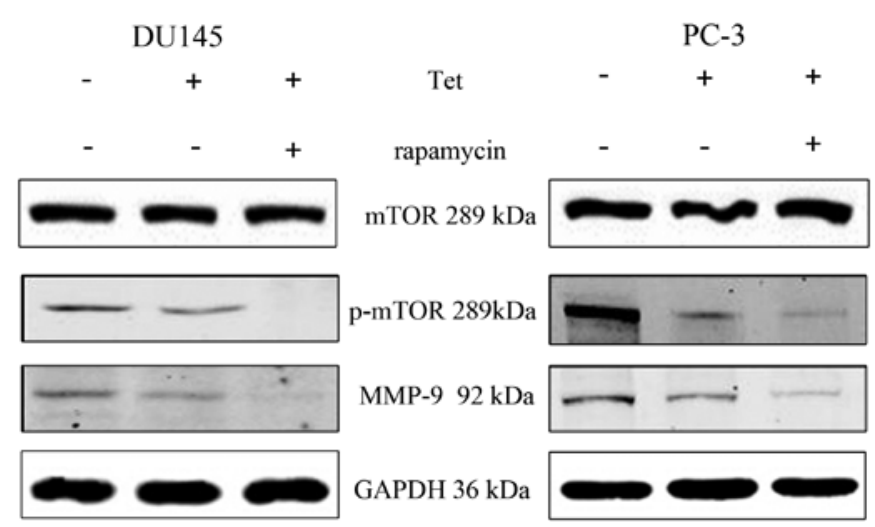

Figure 7. The combined effect of the two agents, tetrandrine and mTOR inhibitor rapamycin, were found to diminish levels of protein MMP-9 more effectively compared with tetrandrine alone. Western blotting was used to detect the levels of mTOR, phospho-mTOR and MMP-9 protein with various treatments in DU145 and PC-3 cells. Representative blots of three experiments are shown.

suggest that, at least partially, the Akt/mTOR/MMP-9 signaling pathway is involved in the tetrandrine-mediated metastatic inhibition of PCa. However, several limitations exist in our study. On the one hand, the lack of animal models in our studies is a short coming, and further considerations should be taken about tetrandrine's function on animal models in our future study. On the other hand, we only observe the anti-metastatic effect of tetrandrine on prostate cancer cells by negatively regulating Akt/mTOR/MMP-9 signaling pathway. Whether alternative signaling pathways participated in this effect of tetrandrine has not yet been elucidated. Hence, it is of necessity for us to resolve these questions in further research.

While our studies have shown that tetrandrine is valid as a single agent for tumor therapy, it might be rational to speculate that it will be used widely in combination with other agents in the clinical setting. Our findings demonstrated that tetrandrine decreased not only p-Akt and p-mTOR activity, but also showed good synergy with LY294002 or rapamycin in the inhibition of PCa cells. Thus, our studies suggest that tetrandrine might be a stronger chemotherapeutic agent when combined with LY294002 or rapamycin.

To the best of our knowledge, our studies provide the first evidence that tetrandrine inhibits PCa cells metastasis by repressing Akt/mTOR and inactivating MMP-9 and that Akt/mTOR appear to be the upstream regulators of MMP-9 inactivation. In conclusion, it is suggested that tetrandrine suppresses metastasis by negatively regulating the Akt/ mTOR/MMP-9 signaling pathway, and that it could serve as a potential inhibitor of tumor metastasis in future therapeutic interventions in PCa patients.

\section{Acknowledgements}

This study was partially supported by the Science and Technology Research Projects of Shaanxi (2014K-11-03-01-03). 


\section{References}

1. Goto Y, Kojima S, Nishikawa R, Enokida H, Chiyomaru T, Kinoshita T, Nakagawa M, Naya Y, Ichikawa T and Seki N: The microRNA-23b/27b/24-1 cluster is a disease progression marker and tumor suppressor in prostate cancer. Oncotarget 5: 7748-7759, 2014.

2. Chaudhary $\mathrm{P}$ and Vishwanatha JK: c-Jun NH2-terminal kinase-induced proteasomal degradation of c-FLIPL/S and $\mathrm{Bcl} 2$ sensitize prostate cancer cells to Fas- and mitochondriamediated apoptosis by tetrandrine. Biochem Pharmacol 91: 457-473, 2014.

3. Lamont KR and Tindall DJ: Minireview: Alternative activation pathways for the androgen receptor in prostate cancer. Mol Endocrinol 25: 897-907, 2011.

4. Shamash J, Dancey G, Barlow C, Wilson P, Ansell W and Oliver RT: Chlorambucil and lomustine (CL56) in absolute hormone refractory prostate cancer: Re-induction of endocrine sensitivity an unexpected finding. Br J Cancer 92: 36-40, 2005.

5. Chen YJ: Potential role of tetrandrine in cancer therapy. Acta Pharmacol Sin 23: 1102-1106, 2002.

6. Pang L and Hoult JR: Cytotoxicity to macrophages of tetrandrine, an antisilicosis alkaloid, accompanied by an overproduction of prostaglandins. Biochem Pharmacol 53: 773-782, 1997.

7. Wu JM, Chen Y, Chen JC, Lin TY and Tseng SH: Tetrandrine induces apoptosis and growth suppression of colon cancer cells in mice. Cancer Lett 287: 187-195, 2010.

8. Wu K, Zhou M, Wu QX, Yuan SX, Wang DX, Jin JL, Huang J, Yang JQ, Sun WJ, Wan LH, et al: The role of IGFBP-5 in mediating the anti-proliferation effect of tetrandrine in human colon cancer cells. Int J Oncol 46: 1205-1213, 2015.

9. Ng LT, Chiang LC, Lin YT and Lin CC: Antiproliferative and apoptotic effects of tetrandrine on different human hepatoma cell lines. Am J Chin Med 34: 125-135, 2006.

10. Li X, Su B, Liu R, Wu D and He D: Tetrandrine induces apoptosis and triggers caspase cascade in human bladder cancer cells. J Surg Res 166: e45-e51, 2011.

11. Lee JH, Kang GH, Kim KC, Kim KM, Park DI, Choi BT, Kang HS, Lee YT and Choi YH: Tetrandrine-induced cell cycle arrest and apoptosis in A549 human lung carcinoma cells. Int J Oncol 21: 1239-1244, 2002.
12. Sun YF and Wink M: Tetrandrine and fangchinoline, bisbenzylisoquinoline alkaloids from Stephania tetrandra can reverse multidrug resistance by inhibiting P-glycoprotein activity in multidrug resistant human cancer cells. Phytomedicine 21: 1110-1119, 2014.

13. Wang TH, Wan JY, Gong X, Li HZ and Cheng Y: Tetrandrine enhances cytotoxicity of cisplatin in human drug-resistant esophageal squamous carcinoma cells by inhibition of multidrug resistance-associated protein 1. Oncol Rep 28: 1681-1686, 2012.

14. Xiao W, Jiang Y, Men Q, Yuan L, Huang Z, Liu T, Li W and Liu X: Tetrandrine induces G1/S cell cycle arrest through the ROS/Akt pathway in EOMA cells and inhibits angiogenesis in vivo. Int J Oncol 46: 360-368, 2015.

15. He BC, Gao JL, Zhang BQ, Luo Q, Shi Q, Kim SH, Huang E, Gao Y, Yang K, Wagner ER, et al: Tetrandrine inhibits Wnt/3catenin signaling and suppresses tumor growth of human colorectal cancer. Mol Pharmacol 79: 211-219, 2011.

16. Qin R, Shen H, Cao Y, Fang Y, Li H, Chen Q and Xu W: Tetrandrine induces mitochondria-mediated apoptosis in human gastric cancer BGC-823 cells. PLoS One 8: e76486, 2013.

17. Toth M, Sohail A and Fridman R: Assessment of gelatinases (MMP-2 and MMP-9) by gelatin zymography. Methods Mol Biol 878: 121-135, 2012

18. Sim HG and Cheng CW: Changing demography of prostate cancer in Asia. Eur J Cancer 41: 834-845, 2005.

19. Gupta GP and Massagué J: Cancer metastasis: Building a framework. Cell 127: 679-695, 2006.

20. Liu C, Gong K, Mao X and $\mathrm{Li} \mathrm{W}$ : Tetrandrine induces apoptosis by activating reactive oxygen species and repressing Akt activity in human hepatocellular carcinoma. Int J Cancer 129: 1519-1531, 2011.

21. Gao JL, Ji X, He TC, Zhang Q, He K, Zhao Y, Chen SH and Lv GY: Tetrandrine suppresses cancer angiogenesis and metastasis in 4T1 tumor bearing mice. Evid Based Complement Alternat Med 2013: 265061, 2013. 